\title{
CONSISTENCY CHeck OF Fuzzy PAIRWISE COMPARISON MATRICES OF DIMENSIONS LARGER THAN 3X3
}

\author{
Zedina Lavic, Branko Vucijak, Mugdim Pasic \& Nedzad Dukic
}
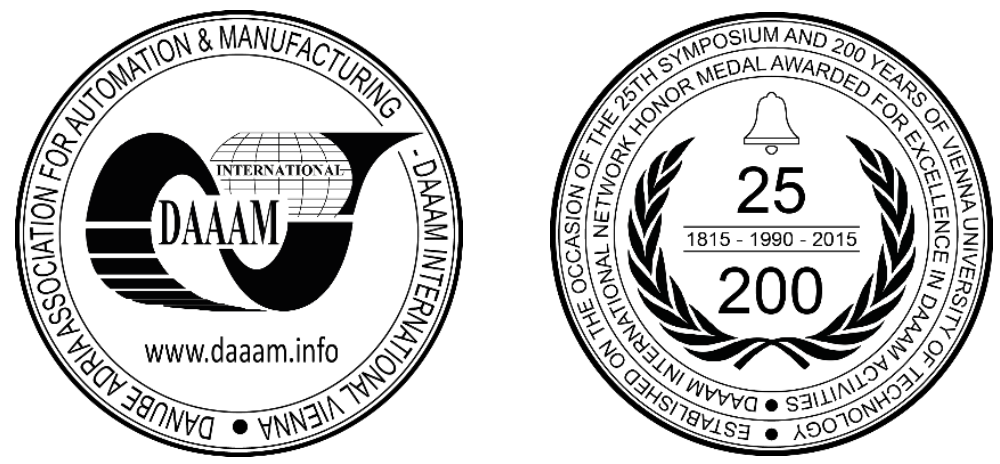

This Publication has to be referred as: Lavic, Z[edina]; Vucijak, B[ranko]; Pasic, M[ugdim] \& Dukic, N[edzad] (2018). Consistency Check of Fuzzy Pairwise Comparison Matrices of Dimensions Larger Than 3x3, Proceedings of the 29th DAAAM International Symposium, pp.0709-0713, B. Katalinic (Ed.), Published by DAAAM International, ISBN 9783-902734-20-4, ISSN 1726-9679, Vienna, Austria

DOI: $10.2507 / 29$ th.daaam.proceedings. 102

\begin{abstract}
In decision-making models based on the Fuzzy Analytic Hierarchy Process, it is necessary to check the consistency of the fuzzy pairwise comparisons matrices. The consistency of the fuzzy matrix $3 \times 3$ can be checked on the basis of the similarity of the fuzzy numbers. In this paper, it is shown how to check the consistency of matrices of larger dimensions by decomposing these matrices to the $3 \times 3$ matrices. The consistency check of fuzzy matrices by decomposition is shown for pairwise comparisons matrices obtained by using fuzzified Saaty's scale. The direction for future research is proposed.
\end{abstract}

Keywords: pairwise comparison matrix; consistency; fuzzy numbers; similarity measure; decomposition

\section{Introduction}

In decision making models based on the Fuzzy Analytic Hierarchy Process (FAHP), the elements of the pairwise comparison matrices are fuzzy numbers. The dimensions of these matrices depend on the number of elements (criteria or alternatives) that are compared with each other in pairs. In most models based on FAHP, the maximal dimension of the matrix is less than or equal to 15 because it is the limit of the classical AHP on which the FAHP is based. As example, in FAHP model for the prioritization of various performance measurement attributes for the performance measurement of employees in an Indian market research firm [1] the largest matrix dimension is 4. A FAHP model for selection of university academic staff [2] used 3 criteria and 3 alternatives i.e. matrices are $3 \times 3$. In the paper [3] for performance evaluation of Turkish cement firms both FAHP and TOPSIS (Technique for Order of Preference by Similarity to Ideal Solution) methods are used. Maximal dimension of pairwise comparison matrices is 5. The approach suggested in [4] is based on FAHP and balanced score card (BSC) for evaluating an information technology department in the manufacturing industry in Taiwan. In FAHP the largest matrix is $4 \mathrm{x} 4$. The largest matrix in FAHP applied to the lead-free equipment selection decision [5] is matrix 7 x 7. In the models above classical matrix consistency imply fuzzy matrix consistency.

Unlike these papers, there are papers in which pairwise comparison matrices dimensions are larger than 15. An example is at [6] where the model for prioritization of human capital measurement indicators using FAHP is developed and numerical application is shown. The greatest matrix dimension is 20, but consistency check is not done. 
For practical problems like this, with more than 15 alternatives or usually conflicting quantitative or qualitative criteria (for example facility layout problem and selection the best among candidates (applicants) for employment), the implication about the consistency of the fuzzy matrix based on the consistency of the corresponding classical matrices cannot be applied.

The purpose of this study is to facilitate the fuzzy matrices consistency check when the matrices dimensions are larger than 3. In the paper [7], the research on fuzzy numbers' similarities is described regarding the consistency check of fuzzy matrix $3 \times 3$. The research was in the function of determining the optimal facilities layout in multi-criteria optimization using fuzzy numbers. The aim of this research is to show that consistency of a fuzzy pairwise comparison matrix of dimension larger than 3, by decomposition, can be checked based on consistency of fuzzy matrix $3 \times 3$. There are no restrictions on this research which would allow the consistency of the fuzzy matrix to be verified on the basis of the fuzzy numbers similarity, without limitation in terms of the matrices dimension.

The similarity measure of fuzzy numbers $A=\left(a^{l}, a^{m}, a^{u}\right)$ and $B=\left(b^{l}, b^{m}, b^{u}\right)$ used in [7] is:

$$
S(A, B)=\frac{1}{1+\left|a^{l}-b^{l}\right|+\left|a^{m}-b^{m}\right|+\left|a^{u}-b^{u}\right|}
$$

Fuzzy numbers are triangular (given in Table 1), obtained by appropriate fuzzification of Saaty's scale [8]. Consistency of a classical matrix implies consistency of the correspondent fuzzy matrix [9].

\begin{tabular}{|c|c|c|}
\hline Definition & Importance intensity & Reciprocals \\
\hline Equal importance & $(1,1,1)$ & $(1,1,1)$ \\
\hline Moderate importance & $(2,3,4)$ & $(1 / 4,1 / 3,1 / 2)$ \\
\hline Strong importance & $(4,5,6)$ & $(1 / 8,1 / 7,1 / 4)$ \\
\hline Very strong importance & $(6,7,8)$ & $(1 / 9,1 / 9,1 / 9)$ \\
\hline Extreme importance & $(9,9,9)$ & $(1 / 3,1 / 2,1),(1 / 5,1 / 4,1 / 3),(1 / 7$, \\
\hline Intermediate values & $(1,2,3),(3,4,5),(5,6,7)$ and & $1 / 6,1 / 5)$ and $(1 / 9,1 / 8,1 / 7)$ \\
\hline
\end{tabular}

Table 1. Fuzzified Saaty's scale

In the work [7], by using Java application, all 3x3 matrices were formed (for the fuzzy scale in Table 1) and the analysis of minimal and mean similarity with respect to the consistency of the corresponding classical matrices is performed. The analyzed matrices are square, positive, and reciprocal:

$$
\left[\begin{array}{ccc}
(1,1,1) & \tilde{a}_{12} & \tilde{a}_{13} \\
1 / \tilde{a}_{12} & (1,1,1) & \tilde{a}_{23} \\
1 / \tilde{a}_{13} & 1 / \tilde{a}_{23} & (1,1,1)
\end{array}\right] .
$$

The analysis of the entire range of minimal values of similarities [0.0041,1] was carried out in [7]. The results are as follows:

- For the minimal similarities lower than 0.0303 there is no consistent classical matrix.

- For some minimal similarities within the range $(0.0303,0.1429)$ there are corresponding average similarities for which both consistent and inconsistent classical matrices exist.

- For some minimal similarities within the range $(0.0303,0.1]$ there are corresponding average similarities for which classical matrices are always consistent.

- When the minimal similarities are higher than 0.1 (except if the minimal similarity is 0.1429 and corresponding average similarity is 0.7873 , when CR of classical matrix is $12.7714 \%$ ). classical matrices are consistent.

- For minimal similarities higher than 0.1429 all the classic matrices are consistent.

The obtained results indicate the possibility of checking the consistency of positive, square, reciprocal matrices of dimensions larger than 3 based on the similarity of the fuzzy numbers.

\section{Decomposition of matrix}

Since the condition of consistency in FAHP is $\tilde{a}_{i k} \otimes \tilde{a}_{k j} \approx \tilde{a}_{i j}$ for all $i, j, k=1,2, \ldots n,[10]$ and for the square, positive, reciprocal matrix of dimension 3 there is only one combination of numbers without repetition $i, j, k=1,2,3$, that a square, positive, reciprocal matrix $3 \times 3$ is a basic element of consistency in FAHP. 
For a positive, reciprocal, square fuzzy matrix of dimension $n$ exists $\left(\begin{array}{l}n \\ 3\end{array}\right)$ a combination without repetition of numbers $i, j, k,(i, j, k=1,2, \ldots n)$ and $\left(\begin{array}{c}n \\ 3\end{array}\right)$ different triplets of elements $\left(\tilde{a}_{i k}, \tilde{a}_{k j} \mathrm{i} \tilde{a}_{i j}\right)$ that are above the diagonal of the matrix dimension $n$. From these different triplets of elements, it is possible to form the same number, i.e. $\left(\begin{array}{l}n \\ 3\end{array}\right)$, square, positive, reciprocal matrices of dimension 3 , that is to decompose the matrix of dimension $n$. For example, for a matrix of dimension 4 exist $\left(\begin{array}{l}4 \\ 3\end{array}\right)=4$ different combinations of numbers $i, j, k=1,2,3,4$ and correspondingly 4 different triplets of elements $\tilde{a}_{i k}, \tilde{a}_{k j}$ i $\tilde{a}_{i j}$ which are above the diagonal of the corresponding square, positive reciprocal matrices of dimension 3 , to which the matrix of the dimension 4 is decomposed. For illustration, the matrix and decomposition of matrix dimension 4 are shown in Table 2 . The triplets of elements that form different matrices $3 \times 3$ are marked bold. These triplets, when aligned by their indexes to the corresponding places in the matrix of dimension 4 , occupy places above the diagonal of the matrix.

In these 4 matrices together $(4 \cdot 27=108$ triplets of elements $)$ all triplets of elements of the matrix $4 \times 4\left(4^{3}=64\right.$ triplets of elements) are contained (with the repetition of the triplets) for which similarity is tested. Therefore, for a matrix of dimension 4, the set of possible values of minimal (but not the mean) similarities is identical to the set of values of possible minimal similarities for the matrices $3 \times 3$. Or, generally, for the matrix of the dimension $n>3$ in which there are in total $n^{3}$ triplets of the elements being tested similarity, the matrices to which it is decomposed together have $\left(\begin{array}{l}n \\ 3\end{array}\right) \cdot 27$ triplets of elements, containing all elements triplets of the matrix of dimension $n$. Therefore, for the matrix of dimension $n$, the set of possible values of the minimal (but not the average) similarities is identical to the set of values of the possible minimal similarities for the matrices of dimension 3. Based on this, and bearing in mind the results of the similarity analysis for the matrices of dimension 3, the conclusion is that examination of the consistency of the matrix of dimension $n$, based on similarity, can be carried out in two ways:

1. by decomposing the matrix of dimension $n$ to square, positive, reciprocal matrices of dimension 3 and

2. based on the minimum similarity for matrices of dimension 3 (without decomposing the matrix of dimension $n$ ).

\begin{tabular}{|c|c|c|c|c|c|c|c|}
\hline & $M_{4 \times 4}=\left[\begin{array}{l}\tilde{a}_{11} \\
\tilde{a}_{21} \\
\tilde{a}_{31} \\
\tilde{a}_{41}\end{array}\right.$ & $\begin{array}{l}\tilde{\boldsymbol{a}}_{12} \\
\tilde{a}_{22} \\
\tilde{a}_{32} \\
\tilde{a}_{42}\end{array}$ & $\begin{array}{l}\widetilde{\boldsymbol{a}}_{13} \\
\widetilde{\boldsymbol{a}}_{23} \\
\tilde{a}_{33} \\
\tilde{a}_{43}\end{array}$ & $\left.\begin{array}{l}\widetilde{\boldsymbol{a}}_{14} \\
\widetilde{\boldsymbol{a}}_{24} \\
\widetilde{\boldsymbol{a}}_{34} \\
\tilde{a}_{44}\end{array}\right]$ & & & \\
\hline$i, j, k$ & $\tilde{a}_{i k}, \tilde{a}_{k j}, \tilde{a}_{i j}$ & \multicolumn{5}{|c|}{$\begin{array}{l}\text { Corresponding square } \\
\text { matrix of dimension } 3\end{array}$} & reciprocal \\
\hline $1,2,3$ & $\tilde{a}_{12}, \tilde{a}_{23}, \tilde{a}_{13}$ & & & $\begin{array}{l}\tilde{a}_{11} \\
\tilde{a}_{21} \\
\tilde{a}_{31}\end{array}$ & $\begin{array}{c}\widetilde{\boldsymbol{a}}_{12} \\
\tilde{a}_{22} \\
\tilde{a}_{32}\end{array}$ & $\left.\begin{array}{c}\widetilde{\boldsymbol{a}}_{13} \\
\widetilde{\boldsymbol{a}}_{23} \\
\tilde{a}_{33}\end{array}\right]$ & \\
\hline $1,2,4$ & $\tilde{a}_{12}, \tilde{a}_{24}, \tilde{a}_{14}$ & & & $\begin{array}{l}\tilde{a}_{11} \\
\tilde{a}_{21} \\
\tilde{a}_{41}\end{array}$ & $\begin{array}{c}\widetilde{\boldsymbol{a}}_{12} \\
\tilde{a}_{22} \\
\tilde{a}_{42}\end{array}$ & $\left.\begin{array}{c}\widetilde{\boldsymbol{a}}_{\mathbf{1 4}} \\
\widetilde{\boldsymbol{a}}_{\mathbf{2 4}} \\
\tilde{a}_{44}\end{array}\right]$ & \\
\hline $1,3,4$ & $\tilde{a}_{13}, \tilde{a}_{34}, \tilde{a}_{14}$ & & & $\begin{array}{l}\tilde{a}_{11} \\
\tilde{a}_{31} \\
\tilde{a}_{41}\end{array}$ & $\begin{array}{c}\widetilde{\boldsymbol{a}}_{13} \\
\tilde{a}_{33} \\
\tilde{a}_{43}\end{array}$ & $\left.\begin{array}{c}\widetilde{\boldsymbol{a}}_{\mathbf{1 4}} \\
\widetilde{\boldsymbol{a}}_{34} \\
\tilde{a}_{44}\end{array}\right]$ & \\
\hline $2,3,4$ & $\tilde{a}_{23}, \tilde{a}_{34}, \tilde{a}_{24}$ & & & $\begin{array}{l}\tilde{a}_{22} \\
\tilde{a}_{32} \\
\tilde{a}_{42}\end{array}$ & $\begin{array}{c}\widetilde{\boldsymbol{a}}_{23} \\
\tilde{a}_{33} \\
\tilde{a}_{43}\end{array}$ & $\left.\begin{array}{c}\widetilde{\boldsymbol{a}}_{24} \\
\widetilde{\boldsymbol{a}}_{34} \\
\tilde{a}_{44}\end{array}\right]$ & \\
\hline
\end{tabular}

Table 2. Decomposition of matrix $4 \times 4$

If the consistency of the fuzzy matrix of dimension $n$ is investigated by decomposing the matrix to $\left(\begin{array}{l}n \\ 3\end{array}\right)$ analogue matrices of dimension 3 , it should be checked that for all these matrices of dimension 3 the corresponding minimal and average similarity take values from the set of values of these indicators obtained by the analysis of the matrices of dimension 3 (when correspondent classical matrices are consistent). If the answer is yes, the fuzzy matrix of the dimension $n$ is consistent. The minimal similarity for the matrix of the dimension $n$ is the minimal value from the set of values of the minimal similarities of matrices of dimension 3 to which the matrix of dimension $n$ is decomposed.

When the consistency of the fuzzy matrix dimension $n$ is checked based on the value of the minimal similarity for that matrix, without decomposing, then the condition of consistency is that the minimal similarity is greater than 0.1429 . 
This is possible because, for the adopted fuzzy scale, all matrices of dimension 3 with a minimal similarity greater than 0.1429 (regardless of the mean similarity) are consistent. This means if the fuzzy matrix of the dimension $n$ satisfies this condition of consistency valid for the fuzzy matrix of dimension 3, this matrix is surely consistent, and the criterion of minimal similarity (greater than 0.1429), can be applied to evaluate the consistency of the square, positive, reciprocal fuzzy matrix of dimension $n$ set to matrices of dimension 3 , without decomposing

\section{Decomposition of a concrete matrix of dimension 4}

Decomposition of concrete matrix of dimensions 4 is shown in Table 3 . The consistency check results for each of these obtained matrices of dimension 3 are shown in Table 4. From the Table 4 it can be seen that the minimal value of the minimal similarity has the matrix $\mathrm{M}_{2}$, i.e. value 0.0526 . As explained above, based on the data in the Table 4 , it is expected that the matrix of dimension 4 will be consistent, that its minimal similarity will be the smallest in the set shown in the Table 4, and that the mean similarity will be smaller than the mean similarity of the individual matrices. After the consistency checking of the matrix dimension 4, it was seen that it is consistent: the values of minimal and mean similarities are 0.05526 and 0.711 respectively, and $C R=0.8719 \%$. Therefore, the minimal similarity value for this matrix is exactly 0.0526 as expected. It is consistent because consistent matrices to which it is decomposed and from which by synthesis, following member indices, can be compiled this matrix of dimension 4 . The value of the mean similarity is less than the mean similarity of the individual matrices.

\begin{tabular}{|c|c|c|c|c|c|}
\hline & $M_{4 \times 4}=\left[\begin{array}{c}(1,1,1 \\
\left(\frac{1}{3}, \frac{1}{2},\right. \\
1 \frac{1}{4}, \frac{1}{3}, \\
\left(\frac{1}{9}, \frac{1}{8},\right.\end{array}\right.$ & $\begin{array}{l}(1,2,3) \\
(1,1,1) \\
\left.\frac{1}{3}, \frac{1}{2}, 1\right) \\
\left(\frac{1}{7}, \frac{1}{6}, \frac{1}{5}\right)\end{array}$ & $\begin{array}{l}(2,3,4) \\
(1,2,3) \\
(1,1,1) \\
\left(\frac{1}{4}, \frac{1}{3}, \frac{1}{2}\right)\end{array}$ & $\left.\begin{array}{l}(7,8,9) \\
(5,6,7) \\
(2,3,4) \\
(1,1,1)\end{array}\right]$ & \\
\hline$i, j, k$ & $\tilde{a}_{i k}, \tilde{a}_{k j}, \tilde{a}_{i j}$ & $\begin{array}{l}\text { The corresp } \\
\text { dimension } 3\end{array}$ & $\begin{array}{l}\text { ponding sc } \\
3\end{array}$ & uare recip & ocal matrix of \\
\hline $1,2,3$ & $\tilde{a}_{12}, \tilde{a}_{23}, \tilde{a}_{13}$ & $M_{1}=$ & {$\left[\begin{array}{l}(1,1,1) \\
\left(\frac{1}{3}, \frac{1}{2}, 1\right) \\
\left(\frac{1}{4}, \frac{1}{3}, \frac{1}{2}\right)\end{array}\right.$} & $\begin{array}{l}(1,2,3) \\
(1,1,1) \\
\left(\frac{1}{3}, \frac{1}{2}, 1\right)\end{array}$ & $\left.\begin{array}{l}(2,3,4) \\
(1,2,3) \\
(1,1,1)\end{array}\right]$ \\
\hline $1,2,4$ & $\tilde{a}_{12}, \tilde{a}_{24}, \tilde{a}_{14}$ & $M_{2}=$ & {$\left[\begin{array}{l}(1,1,1) \\
\left(\frac{1}{3}, \frac{1}{2}, 1\right) \\
\left(\frac{1}{9}, \frac{1}{8}, \frac{1}{7}\right)\end{array}\right.$} & $\begin{array}{l}(1,2,3) \\
(1,1,1) \\
\left(\frac{1}{7}, \frac{1}{6}, \frac{1}{5}\right)\end{array}$ & $\left.\begin{array}{l}(7,8,9) \\
(5,6,7) \\
(1,1,1)\end{array}\right]$ \\
\hline $1,3,4$ & $\tilde{a}_{13}, \tilde{a}_{34}, \tilde{a}_{14}$ & $M_{3}=$ & {$\left[\begin{array}{l}(1,1,1) \\
\left(\frac{1}{4}, \frac{1}{3}, \frac{1}{2}\right) \\
\left(\frac{1}{9}, \frac{1}{8}, \frac{1}{7}\right)\end{array}\right.$} & $\begin{array}{l}(2,3,4) \\
(1,1,1) \\
\left(\frac{1}{4}, \frac{1}{3}, \frac{1}{2}\right)\end{array}$ & $\left.\begin{array}{l}(7,8,9) \\
(2,3,4) \\
(1,1,1)\end{array}\right]$ \\
\hline $2,3,4$ & $\tilde{a}_{23}, \tilde{a}_{34}, \tilde{a}_{24}$ & $M_{4}=$ & {$\left[\begin{array}{l}(1,1,1) \\
\left(\frac{1}{3}, \frac{1}{2}, 1\right) \\
\left(\frac{1}{7}, \frac{1}{6}, \frac{1}{5}\right)\end{array}\right.$} & $\begin{array}{l}(1,2,3) \\
(1,1,1) \\
\left(\frac{1}{4}, \frac{1}{3}, \frac{1}{2}\right)\end{array}$ & $\left.\begin{array}{l}(5,6,7) \\
(2,3,4) \\
(1,1,1)\end{array}\right]$ \\
\hline
\end{tabular}

Table 3. Decomposition of a concrete matrix of dimension 4

\begin{tabular}{|c|r|r|r|r|}
\hline Indexes & Matrix & \multicolumn{1}{|c|}{$\begin{array}{c}\text { Minimal } \\
\text { similarity }\end{array}$} & $\begin{array}{c}\text { Mean } \\
\text { similarity }\end{array}$ & $\begin{array}{c}\text { CR of corresponding } \\
\text { classical matrix } \\
(\%)\end{array}$ \\
\hline $1,2,3$ & $M_{1}$ & 0.125 & 0.7202 & 0.9641 \\
\hline $1,2,4$ & $M_{2}$ & 0.0526 & 0.7731 & 2.2221 \\
\hline $1,3,4$ & $M_{3}$ & 0.0833 & 0.7992 & 0.2096 \\
\hline $2,3,4$ & $M_{4}$ & 0.1111 & 0.7708 & 0.0000 \\
\hline
\end{tabular}

Table 4 . The consistency check results for each of matrices of dimension 3 
It is important to emphasize that neither the first nor the other way have any limitations in terms of the dimension of the matrix. The advantage of the second method is in a simpler algorithm, with fewer calculations, but a smaller set of inputs for which this request is met (a minimum similarity is greater than 0.1429).

Analogue investigations can be carried out for each scale obtained by fuzzification of the Saty's scale in a such way that the elements of the fuzzy scale are fuzzy numbers of the form $\left(a_{i}-t, a_{i}, a_{i}+t\right)$, where $a_{i}$ are the elements of the Saty's scale, and $t$ is any nonnegative real number.

\section{Conclusion}

This paper is focused to the problem of the fuzzy pairwise comparisons matrices consistency check in decision making models based on FAHP. The smallest matrix for which consistency is checked is a positive, square, reciprocal matrix of dimension 3 whose elements are fuzzy numbers. The consistency of this matrix can be checked based on the similarity of the fuzzy numbers. Consistency of analogue matrices of larger dimensions can be checked by decomposing them to matrices of dimension 3. When all matrices of dimension 3 obtained by decomposing are consistent, a decomposed matrix of dimensions larger than 3 is also consistent. This method has no limitations in terms of the dimension of the matrix. Future research can be in the direction of investigating the consistency of the fuzzy matrix whose elements are from different fuzzy scales obtained by fuzzification of the scale suggested by Thomas Saaty.

\section{References}

[1] Aggarwal, R., Singh S. (2013), AHP and Extent Fuzzy AHP Approach for Prioritization of Performance Measurement Attributes, World Academy of Science, Engineering and Technology International Journal of Social, Education, Economics and Management Engineering Vol.7, No.1.

[2] Asuquo, D. E. \& Onuodu, F. E. A (2016), Fuzzy AHP Model for Selection of University Academic Staff. International Journal of Computer Applications 141(1):19-26, May 2016

[3] Ertugrul, I. \& Karakasoglu, N. (2009), Performance evaluation of Turkish cement firms with fuzzy analytic hierarchy process and TOPSIS methods. Expert Systems with Applications, 36(1), 702-715

[4] Lee, H. I., Chen, W. C. \& Chang, C. J. (2008), A fuzzy AHP and BSC approach for evaluating performance of IT department in the manufacturing industry in Taiwan, Expert Systems with Applications, 34(1), 96-107

[5] Tang, Y-C. \& Lin, T.W.(2011) 'Application of the fuzzy analytic hierarchy process to the lead-free equipment selection decision', Int. J. Business and Systems Research, Vol. 5, No. 1, pp.35-56.

[6] Bozbura, F.T., Beskese, A. \& Kahraman, C. (2007), Prioritization of human capital measurement indicators using fuzzy AHP, Expert Systems with Applications, 32(4), 1100-1112.

[7] Lavic, Z, Dukic, N., Pasic, M., \& Vucijak, B. (2016): Research on Similarity Values Relevant for Fuzzy Matrix Consistency Check, Proceedings of the 27th DAAAM International Symposium, B. Katalinic (Ed.), Published by DAAAM International, ISBN 978-3-902734-08-2, ISSN 1726-9679, Vienna, Austria

[8] Saaty, T. L. (1980), Analytic hierarchy process, New York, McGraw-Hill.

[9] Lavic, Z, Pasic, M., Vucijak, B. \& Dukic, N. (2016): Fuzzy Logistics Multicriteria Decision Making Model for Facilities Layout, Proceedings of the 27th DAAAM International Symposium, B. Katalinic (Ed.), Published by DAAAM International, ISBN 978-3-902734-08-2, ISSN 1726-9679, Vienna, Austria

[10] Buckley, J. J. (1985), Fuzzy hierarchical analysis. Fuzzy Sets and Systems, 17(2), 233-247. www.ccsenet.org/mas, Modern Applied Science Vol. 5, No. 4; August 2011. 